Revijalni članak/Review article

\title{
LOW FLOW ANESTHESIA
}

\author{
Cristian Hönemann ${ }^{1}$, Marie-Luise Rübsam ${ }^{1}$
}

${ }^{1}$ Klinikum Leer gGmbH, Klinik für Anästhesie und Intensivmedizin, Augustenstrasse 35-37, 26789 Leer

\section{Abstract}

Historically, rebreathing anesthesia systems were invented to reduce the consumption of volatile anesthetics and to optimize respiratory gas conditioning. However, the industry is still modifying and optimizing the rebreathing circle systems. Most of the modern anesthesia machines today use a rebreathing circle system and are constructed for the use of very low fresh gas flows during inhalational

\section{Introduction}

$\mathrm{H}$ istorically, rebreathing anesthesia systems were invented to reduce the consumption of volatile anesthetics and to optimize respiratory gas conditioning. At the beginning of the $20^{\text {th }}$ century Waters presented his To-and-Fro anesthesia rebreathing system with CO2 absorber (graph 1a). anesthesia. Here we present a practical approach to performing metabolic-flow anesthesia with newer anesthesia agents using pure oxygen as the gas carrier gas.

Keywords: Anesthesia, Inhalation anesthetics, Anesthetic effect, Rebreathing, Pharmacokinetics

In a "dunked dog experiment" (graph 1b). Guedel suggested the use of rebreathing systems to save volatile anesthetics and optimize breathing gas humidity and temperature. At the ASA meeting he demonstrated its use. This system was further developed by Draeger ${ }^{\oplus}$ Lübeck and other companies. This is not common knowledge. However, industry is still modifying and optimizing the rebreath-

Graph 1a: Ralph Waters To-and-Fro System 1924

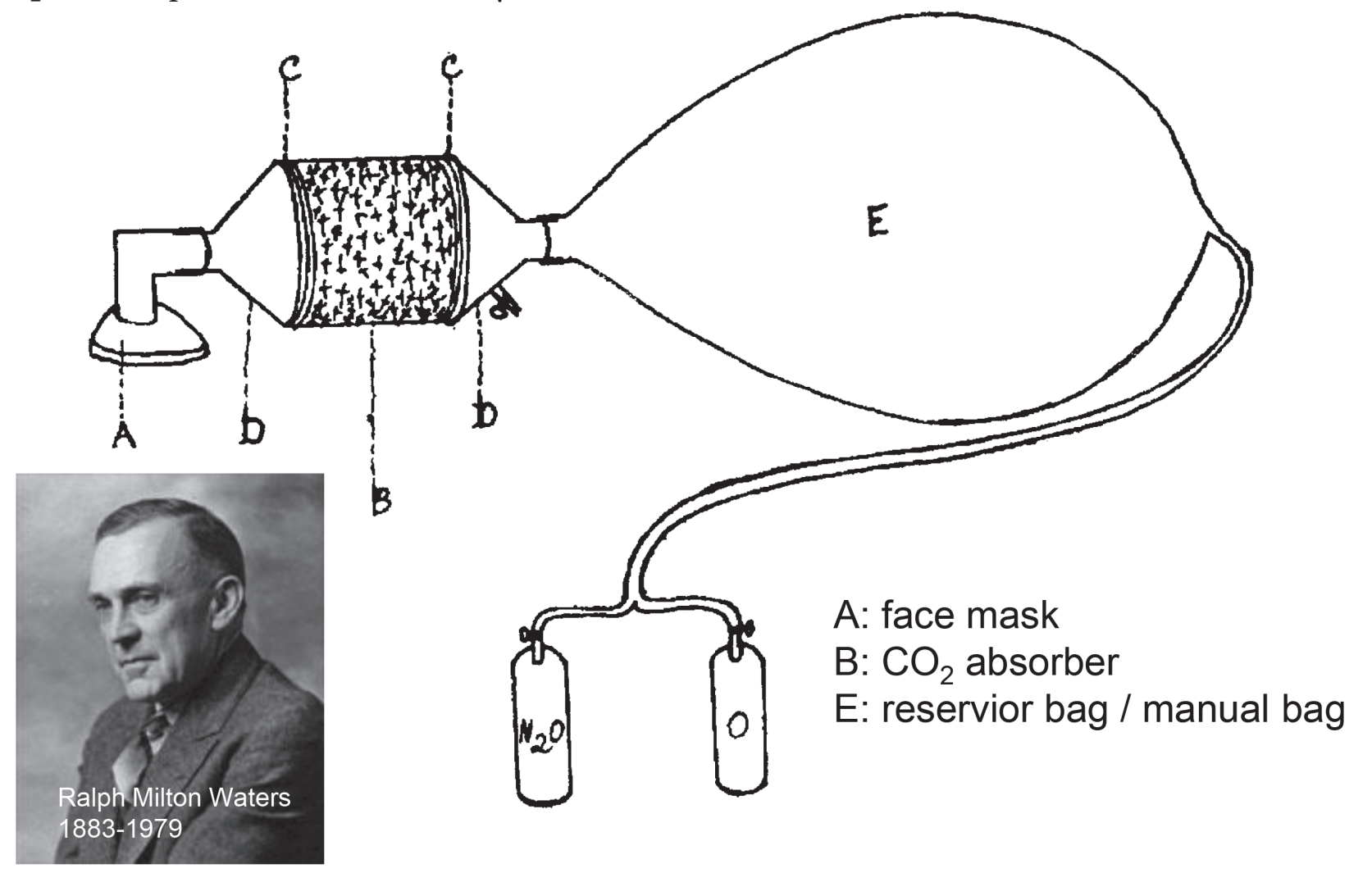

Corresponding author: Christian Hönemann Klinikum Leer gGmbH; Klinik für Anästhesie und Intensivmedizin Augustenstrasse 35-37 26789 Leer; Email: christian.hoenemann@klinikum-leer.de; Phone: +49 (0) 15208961106 
Graph 1b: Guedel's dunked dog experiment at the ASA meeting

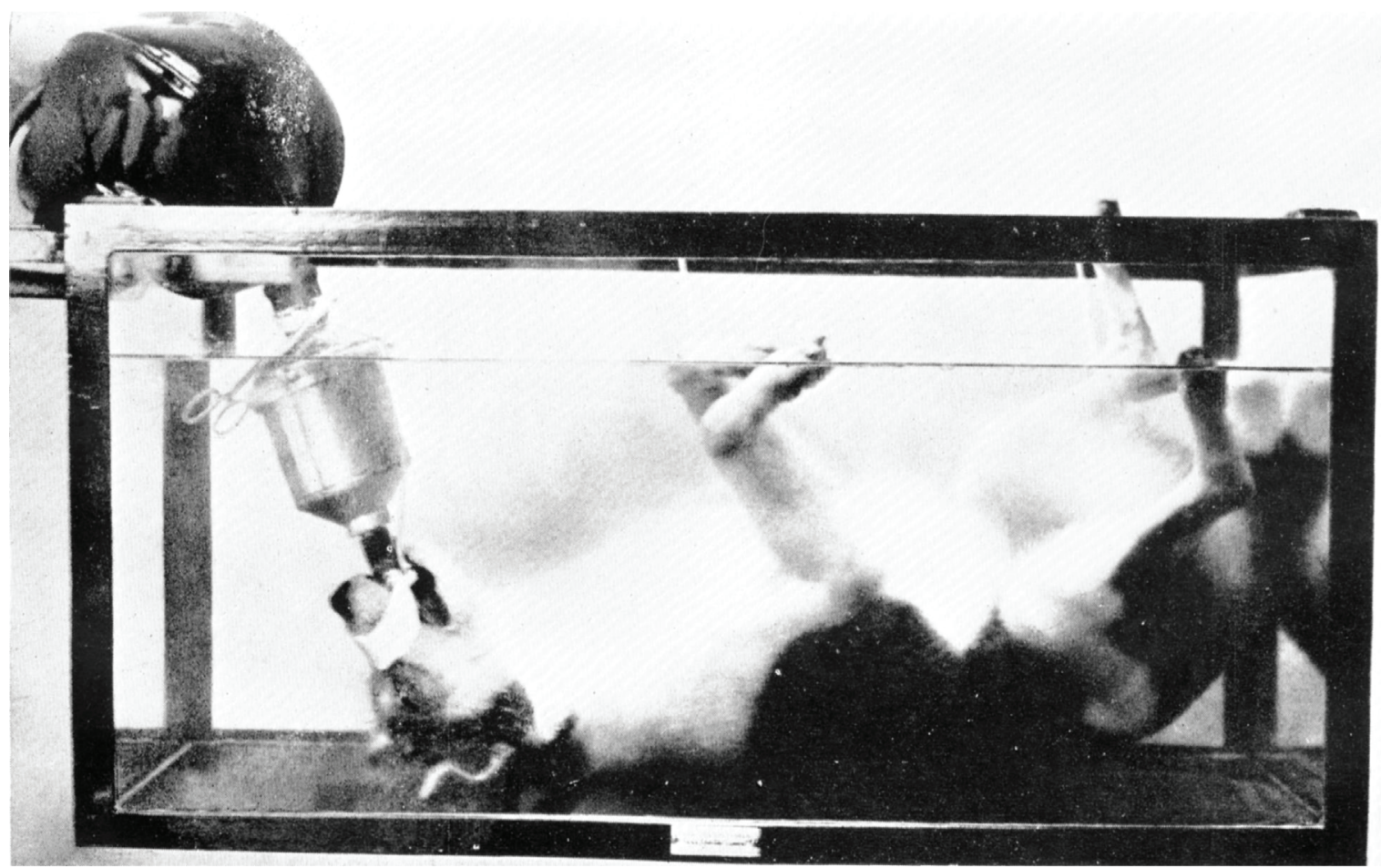

ing circle systems. Most of the modern anesthesia machines today use a rebreathing circle system and are constructed for the use of very low fresh gas flows during inhalational anesthesia.

\section{The use and construction of rebreathing systems}

In semi-closed and closed rebreathing systems, there is a correlation between fresh gas flow, temperature and humidity: The lower the fresh gas flow used, the higher the temperature and humidity of inhaled anesthetic gases is. The lower the fresh gas flow used, the more surplus gas volume is decreased. Low surplus volume of anesthetic gases results in lower cost (up to $85 \%$ savings) for volatile anesthetic agents, decreased environmental pollution, reduced ozone layer depletion and consequently a lower impact on climate change (less greenhouse effects).

Different low flow anesthesia techniques are defined with the use of semi closed anesthesia rebreathing systems. Low flow anesthesia (fresh gas flow $1 \mathrm{~L} / \mathrm{min}$ ), minimal flow anesthesia (fresh gas flow $0.5 \mathrm{~L} / \mathrm{min}$ ), metabolic flow anesthesia (fresh gas flow $0,25-0,35 \mathrm{~L} / \mathrm{min}$ ) and non-quantitative anesthesia in the almost closed circuit anesthesia (patient uptake $=$ fresh gas flow).

In some anesthesia systems fresh gas flows and volatile anesthetic concentration can be controlled automatically. This is called quantitative closed circuit anesthesia (closed loop automatic control of volatile anesthetics concentration and anesthetic gas composition, e.g. Zeus ${ }^{\circledast}$ Draeger, Lübeck, Germany).

The manufacturer constructed valve controlled rebreathing circuits, e.g. ISO ${ }^{\circledR}$ rebreathing circuit system (graph 2). Here the exhaled breathing gases are stored during exhalation in the manual-bag, which is then used as a reservoir bag. During the next breathing cycle, the exhaled air is taken out of the reservoir bag, mixed with fresh gas during inspiration. The air flows through the $\mathrm{CO} 2$ absorber. Here, due to an exothermic chemical reaction, $\mathrm{CO} 2$ is chemically bound and water, as well as heat, is introduced to the inspired gases (graph. 3).

Basically, all modern anesthesia-rebreathing circuits follow this concept. Nowadays optimization of rebreathing circuits is targeting increase of the system's "speed", in order to change fresh gas composition. This target can be achieved passively, e.g. reduction of system volume, or actively e.g. flow within the circle mixing the gas in the system. Apart from optimizing the breathing system technology, vaporizers with a high maximum dosage can help increasing the speed of the system as well.

The "speed" is a very important aspect when talking about low flow anesthesia. Slow breathing systems imply less controllability. Example: Once 
Graph 2: Gauss und Wieland 1925 ISO 8 - "Narcylennarkoseapparat"

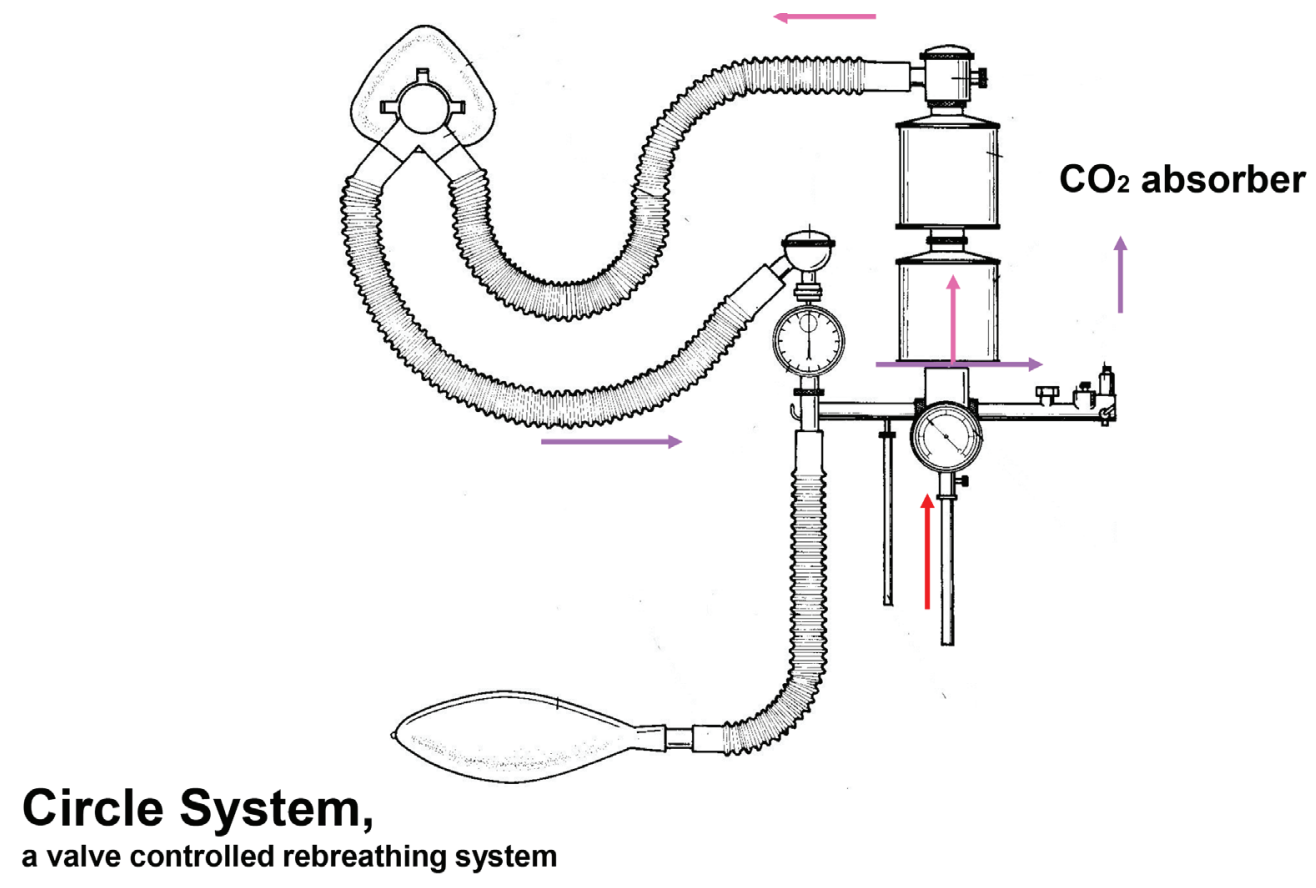

Graph 3: Chemical reaction and Carbon dioxide absorption

$$
\mathrm{CO}_{2}+\mathrm{Ca}(\mathrm{OH})_{2} \rightarrow \mathrm{CaCO}_{3}+\mathrm{H}_{2} \mathrm{O}+\mathrm{dT}
$$

you intend to increase the anesthetic gas concentration, a slow breathing system will take several minutes until the targeted concentration is reached. The lower the flow, the longer the time constant. A low system volume in the breathing system and higher maximum dosages of vaporizers can reduce this time constant significantly. This is worth looking at, as low flow anesthesia provides valuable advantages.

\section{Why should we perform low flow anesthesia?}

(1) Most important reasons to reduce the fresh gas flow are medical reasons. During high flow anesthesia decompressed, dry and cool fresh gas released into the system results with low humidity and temperature of the inhaled gas. Even after a short time, dry and cold inhaled gas may lead to an impairment of the muco-ciliary clearance and pulmonary function and finally, pulmonary complications during the perioperative period (e.g. due to atelectasis, pneumonia, bronchitis). Low flow anaesthesia ensures good breathing gas conditioning (humidity and temperature) and therefore contributes to the pre-

\section{dT=heat}

vention of lung damage and efforts to maintain core body temperature. For this reason, the deployment of low flow anesthesia for short surgical procedures with a minimum 15-minute duration is already recommended. (1)

(2) Ecological reasons: The effects of anesthetic gases on the environment are significant because they contribute to the greenhouse effect and to global climate change. The environmental chemist and Nobel Peace Prize Laureate, Ole Jon Nielsen (Copenhagen University) researched these effects and proved that anaesthetic gases remain in the atmosphere for several years. According to his projections, the harmful effects of anesthetic gases worldwide per year equals a $\mathrm{CO}_{2}$ emissions of up to $1,000,000$ cars for the same time period. (2)

(3) Economical reasons: The consumption of anesthetic gases in hospitals presents a significant cost factor. But not only do the anesthetic agents themselves contribute to the costs, carrier gases e.g. oxygen or medical air, which are 
Graph 4: Modern circle system of the Primus/Apollo family (Draeger ${ }^{\circledR}$ )

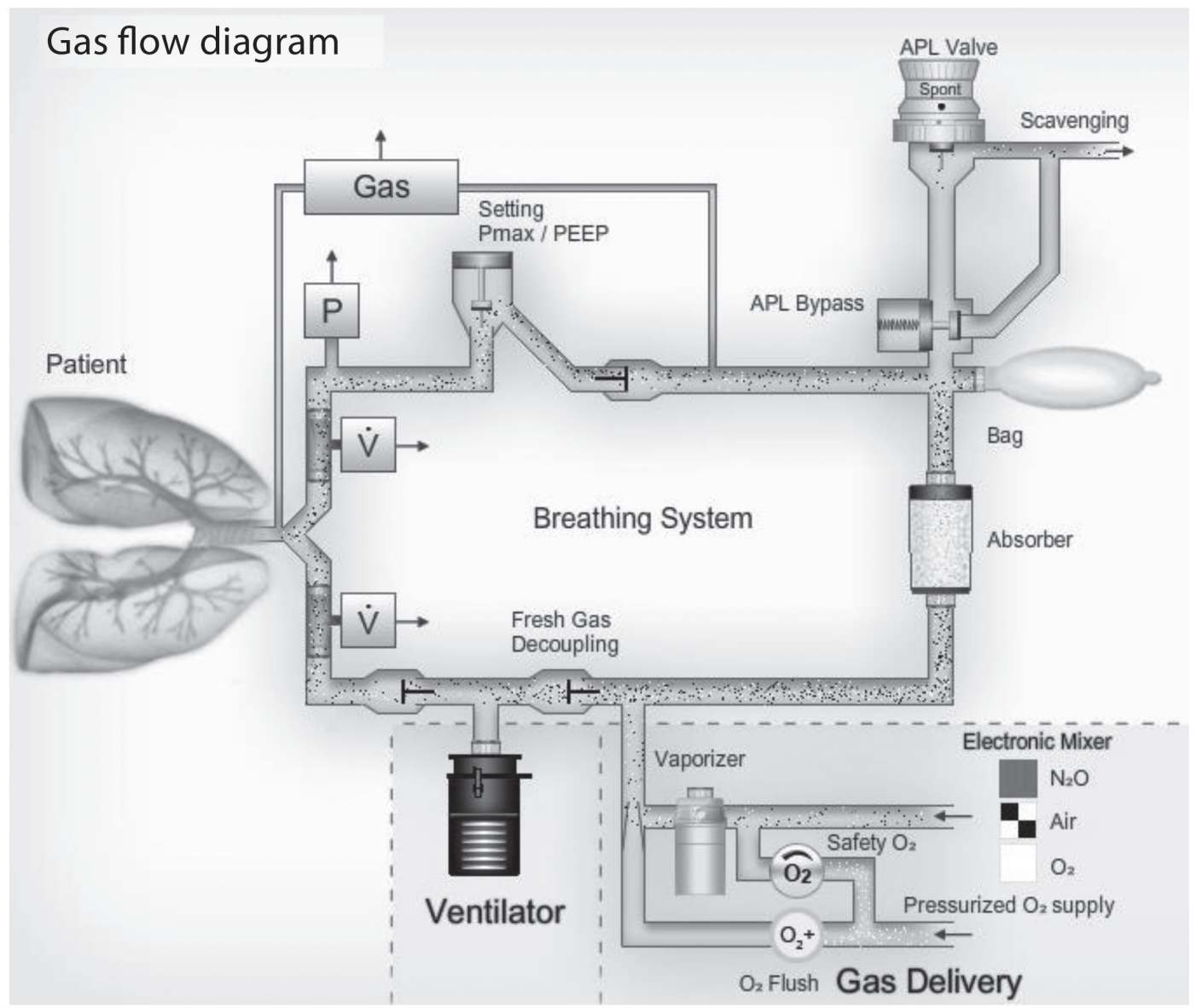

disposed of unused in equal measures, also increase the costs. Depending on the size of the hospital, the potential for savings in pounds amounts to five digit sums per year. (1)

(4) Pharmacokinetical reasons: The metabolism of the most currently used anesthesia gases is very low. This, in combination with their low solubility, results in relatively high saturation in the central compartment and a lower saturation in the peripheral compartments. Consequently, the influx and wash-out is fast, resulting in a very good controllability. Ideal volatile anesthetic gases are Sevoflurane and Desflurane, as they show the lowest solubility.

Table 1. ]Some general rules for low flow anesthesia

(1) Lower the fresh gas flow, better the gas conditioning (positive influence on mucociliary clearance)

(2) Lower the fresh gas flow, lower the gas waste and negative impact on the environment

(3) Higher the system volume, slower are the changes in the system; smaller the system volume, faster are the changes.

(4) Higher the fresh gas flow, quicker is the system; lower fresh gas flow, slower is the system.

(5) Higher the maximum dosage of the vaporizer, faster are the anaesthetic gas changes in the system

(6) Choice of anesthetic gas influences the controllability due to improved pharmacokinetics

\section{How to perform Minimal-flow anaesthesia with oxygen as a carrier gas?}

Recommendations of the World Health Organization for the prevention of surgical infections

For the induction of anesthesia, a pre-oxygenation with $100 \%$ oxygen at $6 \mathrm{~L} / \mathrm{min}$ for 1 to $3 \mathrm{~min}$ utes is still recommended.

After the intravenous or inhalational induction, analgesia and muscle relaxation is established for tracheal intubation. Since nitrous oxide is not in use, there might be a need for $20 \%$ higher dosing of opioids. After endotracheal intubation or insertion of a laryngeal mask, the patient is connected to the circuit system. 
For a time period of 1 to 8 minutes the fresh gas flow settings must consist of $100 \%$ oxygen with a $0.5-1 \mathrm{~L} / \mathrm{min}$. The vaporizer setting for isoflurane should be 5 to 6 vol\%, for sevoflurane 5 to $6 \mathrm{vol} \%$ and for desflurane $12 \mathrm{vol} \%$. The inspiratory oxygen concentration will stabilize in the range of 60 through $80 \mathrm{vol} \%$ depending on the age and weight of the patient, as well as the anesthesia machine used. However, the oxygen concentration must be adapted for individual cases. According to the patient response, the performance characteristics of the machines used and the surgical requirements vary.

Once the target MAC value of 0.8 to 1 has been reached, a reduction of fresh gas flow with $100 \%$ oxygen to 0.25 to $0.35 \mathrm{~L} / \mathrm{min}$ is recommended. During this period the anaesthetic agent concentration setting needs to be adjusted: Isoflurane to 4 to 6 vol\%, sevoflurane 4 to $6 \mathrm{vol} \%$ and desflurane 8-12 vol\% according to the MAC value measured within the circle system.

During metabolic flow anesthesia, monitoring is necessary: Inspiratory oxygen concentration with a lower alarm threshold of at least 28 vol\% and minute volume ventilation set for the lower alarm threshold of $0.5 \mathrm{~L} / \mathrm{min}$ below desired target.

The anaesthetic agent concentration in the breathing system also needs to be measured. Upper limits for isoflurane should be set to 2 to 2.5 vol\%, for sevoflurane to 3 to $3.5 \mathrm{vol} \%$ and for desflurane to 8 to $10 \mathrm{vol} \%$.

It might be helpful to use prediction software for the oxygen and volatile anesthetic concentration (e.g. Dräger SmartPilot ${ }^{\oplus}$ )

For the reversal of anesthesia the vaporizer setting has to be reduced to $0 \%$. This can be done approximately 10 to 15 minutes prior to the end of the operation. During this period low flow of $0.35 \mathrm{~L} /$ min is still used. After the patient is switched from mechanical ventilation to spontaneous breathing, following the completion of suture, prior to extubation, the anesthetist washes out the volatile anesthetics out of the anesthesia circuit system with $100 \%$ oxygen at $6 \mathrm{~L} / \mathrm{min}$..

\section{Practical hints and pitfalls}

Using very low fresh gas flow results with a very long time constant. This means that changes of the vaporizer dial will lead to very slow changes of the anesthetic agent concentrations within the circuit. If the anesthesiologist wants to increase anaesthetic agent concentration using this long time constant, the fresh gas flow remains unchanged at a very low level of $0.35 \mathrm{~L} / \mathrm{min}$ and the vaporizer settings have to be increased to maximum output. However, deepening the anaesthesia with isoflurane alone, needs to be established at maximum isoflurane vaporizer output, while simultaneously increasing the fresh gas flow up to $1 \mathrm{~L} / \mathrm{min}$, until reaching the new end-tidal MAC value. Once the desired concentration is reached, the vaporizer should be set back to isoflurane maximum output of 5 or 6 vol\%. For sevoflurane and desflurane vaporizers should be set at 4 to 7 vol\% and 12 to 14 vol\% respectively. Reducing the anaesthetic agent concentration using the long time constant is very similar. The fresh gas flow remains unchanged at $0.35 \mathrm{~L} / \mathrm{min}$ with the closed vaporizer; shortly before the desired concentration is reached, the vaporizer should be set back to the adequate concentrations: isoflurane 4.5 vol\%, sevoflurane 4.5 to $5 \mathrm{vol} \%$ and desflurane 8 to 12 vol\%.

Rapid changes of depth of anaesthesia using a short time constant is also possible. Here the anesthetist has to increase the fresh gas flow to $4 \mathrm{~L} / \mathrm{min}, 100 \%$ oxygen (Warning: adjust/reduce vaporizer setting if necessary). Once the desired anaesthetic agent concentration is reached-as a rule: after 1 to 3 minutes - the fresh gas flow is reduced again to $0.35 \mathrm{~L} / \mathrm{min}$ or the vaporizer setting to the wanted concentration of the volatile anesthetic.

For every low-flow anaesthesia, measuring the expiratory end-tidal anaesthetic agent concentration in the breathing system is mandatory. As an alternative to short-term deepening of the anaesthesia by increasing fresh gas flow, additional intravenous injection of the hypnotic agent or analgetic should be considered.

\section{Use of pure oxygen}

In general, metabolic-flow anaesthesia, as recommended by the $\mathrm{WHO}$, is the consequence of using minimal-flow anaesthesia and omitting an oxygen/nitrous oxide or oxygen/air mixture. By using pure oxygen as the carrier gas, denitrogenation can be omitted at the start of inhalational an- 
aesthesia because nitrous oxide does not have to be washed in. The advantages of rebreathing systems can therefore be used right from the start. An initially high fresh gas flow is only briefly needed or not at all. The vaporizer output for isoflurane is 5 to $6 \%$ (depending on manufacturer and model year), $8 \%$ for sevoflurane and $18 \%$ for desflurane. Following pre-oxygenation, a low fresh gas flow can be selected at the start of inhalational anaesthesia. The time estimated for acheiving an adequate anaesthesia gas concentration determines the level of fresh gas flow. The higher the fresh gas flow, the more rapidly the desired anaesthesia gas concentration is achieved in the rebreathing system. The lower the fresh gas flow, the slower the volatile anaesthetic agent concentration increases. From our clinical experience we recommend a fresh gas flow of 0.5 to $1 \mathrm{~L} / \mathrm{min}$ in order to reach an adequate anaesthetic agent concentration in the circuit system. This allows reaching a MAC value of 0.9 within 5 to 7 minutes for any modern anaesthetic agent. This timeframe is indicated because by administering an intravenous hypnotic (thiopental, propofol) for 7 to 9 minutes an adequate depth of hypnosis is induced. As the plasma concentration of the hypnotics falls, the concentration of the inhalational anaesthetics increases.

Modern anaesthesia agents are metabolised to only minor extent, have low solubility and are therefore easy to control. As a result, the time for washing in the anaesthesia gases into the circuit system is defined in a simplified way by the following factors: for the most it depends on the volume of the circuit system and the maximum vaporizer output, as well as on the patient's lung capacity and body weight. This fact is confirmed by studies concerning the influx rates of the anaesthesia gases sevoflurane and desflurane in Dräger anaesthesia machines Primus and Zeus. The rates od fresh gas flow only differ slightly and are not clinically relevant (fresh gas flow $0.5 \mathrm{~L} / \mathrm{min}$ to $1 \mathrm{~L} / \mathrm{min}$ for influx). For isoflurane, wash in of the anesthetic can only be achieved with a fresh gas flow of $1 \mathrm{~L} /$ $\min$.

Once the anaesthesia gas concentration in the circuit system has reached the desired value (e.g., MAC 0.9), the fresh gas flow can be further reduced (e.g., from 0.5 to $0.35 \mathrm{~L} / \mathrm{min}, 100 \% \mathrm{O} 2$ ). This procedure enables adequate use of the rebreathing system. As in the case of minimal-flow anaesthesia, the vaporizer can be shut off roughly 10 minutes before the end of the operation. At the same time as the last suture is performed, the fresh gas flow is increased to 4 to $6 \mathrm{~L} / \mathrm{min}$ in order to wash out the anaesthesia gas. An even higher fresh gas flow in the reversal phase does not wash out the anaesthesia gas more rapidly because with a fresh gas flow of 4 to $6 \mathrm{~L} / \mathrm{min}$, the rebreathing portion is already below $2.5 \%$. Increasing the fresh gas flow three-fold to $18 \mathrm{~L} / \mathrm{min}$ reduces rebreathing from 2.5 to only roughly $0.75 \%$ and will clinically cause only minor acceleration of reversal. With metabolic-flow anaesthesia, despite the significant reduction in fresh gas flow, a certain excess volume of gas and maintaining a standardised fresh gas composition are not omitted.

This means yet another distinct simplification of low- and minimal-flow anaesthesia when using pure oxygen as carrier gas compared with low- and minimal-flow anaesthesia using oxygen/air mixture or oxygen/nitrous oxide mixture as carrier gas.

\section{SCHEMATIC PROCEDURE, PATIENT \\ AGE OF > 6 MONTHS}

\section{Premedication}

Premedication according to usual model

\section{Induction}

- Pre-oxygenation with 100\% oxygen at $6 \mathrm{~L} /$ min for 1 to 3 minutes, with a face mask

- Intravenous administration of the hypnotic or inhalation induction

- Analgesia and relaxation (attention: There might be a need for $20 \%$ higher dosing of induction opioid)

- Endotracheal intubation or insertion of a larynx mask

- Connection of patient to the circuit system

\section{Initial phase}

- Duration 1 to 8 minutes-fresh gas flow settings: $100 \%$ oxygen $1 \mathrm{~L} / \mathrm{min}$,

- Vaporizer settings:

Isoflurane 5 to 6 vol\%

Sevoflurane 5 to 6 vol\%

Desflurane 12 vol\%

- The inspiratory oxygen concentration will stabilize between 60 and $80 \mathrm{vol} \%$ depending on age and weight. 
* All values are clinically tested orientation values. However, in individual cases they

must be adapted according to the individual response by the patient, the performance

characteristics of the machines used and the operational requirements.

Once the target MAC value of 0.8 to 1 has been reached

- Reduction of fresh gas flow for $100 \%$ oxygen to 0.25 to $0.35 \mathrm{~L} / \mathrm{min}$

- Change of anaesthetic agent concentrationsee practical hints

\section{Monitoring}

- Inspiratory oxygen concentration with a lower alarm threshold of at least $28 \mathrm{vol} \%$.

- Minute volume: set lower alarm threshold to $0.5 \mathrm{~L} / \mathrm{min}$ below the desired target value.

- Monitoring the anaesthetic agent concentration in the breathing system: set upper limits for isoflurane to 2 to 2.5 vol\% for sevoflurane to 3 to $3.5 \mathrm{vol} \%$, and for desflurane to 8 to $10 \mathrm{vol} \%$.

\section{Reversal}

- Reduction of vaporizer setting to $0 \%$ approximately 10 to 15 minutes prior to the end of the operation.

- Maintaining low fresh gas flow of $0.35 \mathrm{~L} / \mathrm{min}$.

- Switching of patient to spontaneous breathing.

- Following completion of suture, prior to extubation: purging of the system with $100 \%$ oxygen at $6 \mathrm{~L} / \mathrm{min}$.

- Postoperative care of the patient according to the usual internal procedures of the department.

\section{Practical hints}

Increasing the anaesthetic agent concentration using the long time constant $\min$.

- Fresh gas flow remains unchanged at $0.35 \mathrm{~L} /$

- Increase the vaporizer settings to maximum output. Special feature of isoflurane: deepening of the anaesthesia with isoflurane alone should only be established at maximum isoflurane vaporizer output, while simultaneously increasing the fresh gas flow.

- Once the desired concentration is reached, set the vaporizer to:
Isoflurane maximum output to 5 or 6 vol\%

Sevoflurane to 5 to 7 vol\%

Desflurane to 12 to $14 \mathrm{vol} \%$

Reducing the anaesthetic agent concentration using the long time constant

- The fresh gas flow remains unchanged at 0.35 $\mathrm{L} / \mathrm{min}$.

- Close the vaporizer; shortly before the desired low concentration is reached, set the vaporizer to:

Isoflurane $4.5 \mathrm{vol} \%$

Sevoflurane 4.5 to 5 vol\%

Desflurane 8 to 12 vol\%

Rapid change of anaesthesia depth using a short time constant

- Increase the fresh gas flow to $4 \mathrm{~L} / \mathrm{min}, 100 \%$ oxygen

(Warning: adjust/reduce vaporizer setting if necessary).

- Once the desired anaesthetic agent concentration is reached-as a rule

after 1 to 3 minutes-reduce the fresh gas flow again to $0.35 \mathrm{~L} / \mathrm{min}$.

- Set vaporizer to:

Isoflurane maximum output: 5 or 6 vol\%

Sevoflurane 4.5 to 5 vol\%

Desflurane 8 to 12 vol\% $60 \mid 61$

- For all low-flow anaesthesia, measuring the expiratory end-tidal anaesthetic agent concentration in the breathing system is mandatory.

As an alternative to short-term deepening of the anaesthesia by

increasing fresh gas flow, additional intravenous injection of the

hypnotic or analgesic should be considered.

Warning-inspiratory $\mathrm{O} 2$ alarm

- If with the chosen setting, the inspiratory oxygen concentration drops to less than $28 \mathrm{vol} \%$, increase the fresh gas flow of oxygen to $0.5 \mathrm{~L} / \mathrm{min}$.

- Check the system for leakages

- Check the plausibility of the oxygen measurement

Warning-fresh gas flow too low

- The minute volume drops, airway peak pressure drops, the machine sounds fresh gas alarm, the reservoir bag collapses.

- Fill the breathing system by increasing the fresh gas flow to $2 \mathrm{~L} / \mathrm{min}$ for roughly one minute.

- Search for leakage (hole in hose system, bag valve mask, CO 2 absorber 
correctly secured?). If the leakage cannot be repaired, increase the fresh gas flow with $100 \%$ oxygen by $0.5 \mathrm{~L} / \mathrm{min}$ and switch to minimal-flow or low-flow anaesthesia at $1 \mathrm{~L} / \mathrm{min}$.

Fresh soda lime is essential

- Observe the inspiratory CO 2 concentration and the soda lime. If the inspiratory $\mathrm{CO} 2$ concentration increases, this is an indication that the soda lime should be replaced

\section{References}

1. http://www.draeger.com/sites/assets/PublishingImages/Generic/UK/Booklets/low-minimal-flow-anaesthesie-bk-9067990-en.pdf

2. Honemann C, Hagemann O, Doll D. Inhalational anaesthesia with low fresh gas flow. Indian J Anaesth 2013;57:345-50. 